\title{
Evaluating polymeric sampling as a tool for predicting the bioaccumulation of polychlorinated biphenyls by fish and shellfish
}

Stine N. Schmidt ${ }^{1 *}$ and Robert M. Burgess ${ }^{2}$

${ }^{1}$ National Research Council, US Environmental Protection Agency, Office of Research and Development, Atlantic Coastal Environmental Sciences Division, Narragansett, Rhode Island, USA and ' US Environmental Protection Agency, Office of Research and Development, Atlantic Coastal Environmental Sciences Division, Narragansett, Rhode Island, USA. $\left({ }^{*}\right)$ Corresponding author; Stine N. Schmidt, stnsc@mst.dk, phone: +45 20462089. Current affiliation: Danish Environmental Protection Agency, Centre for Safe Chemistry, Unit for Pesticides \& Biocides, Odense, Denmark.

\section{Content of the Supporting Information}

- Description of the polymeric sampling approaches equilibrium sampling and passive sampling.

- Figure S1: Illustration of the concept of the review.

- Details on the literature search.

- Table S1: Literature search terms as entered into Web of Science.

- Table S2: Overview of the 13 peer-reviewed scientific publications and selected experimental details.

- Table S3: Summary of the polymeric sampling data across the 13 peer-reviewed scientific publications.

- Table S4: Summary of the bioaccumulation data across the 13 peer-reviewed scientific publications.

- Figure S2: Data and log-log linear regressions for each of the 144 individual organism data sets.

- Table S5: Selected details on the input data and numerical information on the 144 regressions pro

- Assessment of the semipermeable membrane device (SPMD) subdata set.

- Table S6: Statistical analysis of five key experimental variables for sediment-deployed samplers.

- Table S7: Statistical analysis of five key experimental variables for water column-deployed samplers.

- Details on the five key experimental variables.

- References. 


\section{Description of the polymeric sampling approaches equilibrium sampling and passive sampling}

\section{Equilibrium sampling}

The first approach, often called equilibrium sampling, is used to estimate the exposure of hydrophobic organic contaminants (HOCs) in sediment interstitial water.

Following this approach, a very thin polymer (often between $1-10 \mu \mathrm{m}$ ) is fully equilibrated with the sediment sample. ${ }^{1-4}$ To reach equilibrium between the sediment interstitial water and the polymer for highly hydrophobic compounds (e.g., HOCs with $\log \mathrm{K}_{\mathrm{ow}}>5-6$ ), the polymer is incubated with the sediment sample in the laboratory (ex situ) for approximately 1-2 weeks with agitation/ tumbling. ${ }^{3,4}$

After incubation, the concentration in the polymer at equilibrium with the sediment (i.e., the equilibrium polymer concentration, $C_{P}$ ) is confirmed using either samplers of multiple polymer thicknesses or time series measurements. ${ }^{4,5}$

\section{Passive sampling}

The second approach, often called passive sampling, is used to estimate the exposure of HOCs in sediment interstitial water and in the water column. ${ }^{6,7}$

Following this approach, a thicker polymer (often $\geq 25 \mu \mathrm{m}$ ) is placed within the sediment or in the water column on site (either in situ or in an experimental laboratory system mimicking in situ conditions) and deployed for approximately one month under ambient conditions. ${ }^{8}$ Passive sampling deployments can also be performed under ex situ conditions with agitation/ tumbling. ${ }^{6}$ Less hydrophobic compounds (e.g., HOCs with log $\mathrm{K}_{\text {ow }}<3-4$ ) may reach equilibrium between the environmental sample and the polymer, whereas this is rarely achieved for highly hydrophobic compounds. ${ }^{9}$

After deployment and recovery, the concentration in the polymer is measured and assumed to reflect equilibrium conditions or equilibrium concentrations are inferred using (i) performance reference compounds (PRCs) amended into the polymer before deployment, (ii) time series measurements, or (iii) polymer thickness measurements. ${ }^{10}$ 

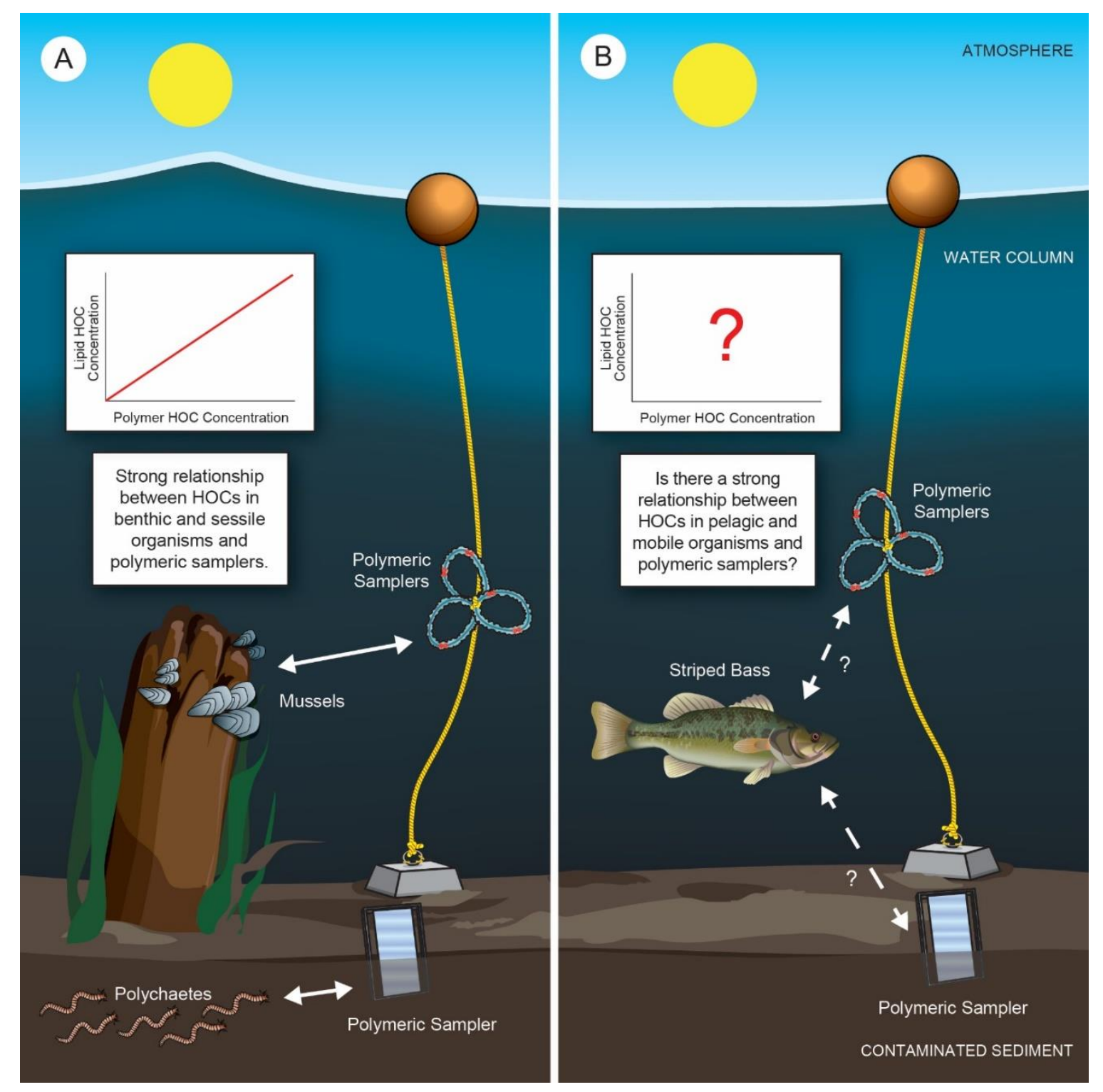

Figure S1. Illustration of the (A) established correlative and significant relationship between bioaccumulation by benthic and sessile invertebrates and accumulation in polymeric samplers and (B) unknown relationship between bioaccumulation by pelagic and mobile fish and shellfish and accumulation in polymeric samplers (as evaluated in this review). The dashed lines labelled with question marks indicate the uncertainty in our understanding of the role of the exposure routes and the relationships between the concentrations in the fish/shellfish and the polymeric samplers. 


\section{Details on the literature search}

A literature search was conducted via the online database Web of Science to identify peer-reviewed scientific publications reporting (i) experimental polymeric sampling data on polychlorinated biphenyls (PCBs) in sediment interstitial water and/or in the water column as well as (ii) experimental data or monitoring data on bioaccumulation of PCBs by fish and shellfish. More specifically, the literature search sought to retrieve corresponding data on PCB equilibrium polymer concentrations and lipid-normalized concentrations of the same PCBs in fish and shellfish. The literature search was not constrained to retrieving only studies on PCBs but more broadly on a range of HOCs. The literature search was performed several times (last time in November 2019). The literature search was based on three common denominations of polymeric sampling; passive sampling, equilibrium sampling, and polymeric sampling. The three denominations were each combined with additional search terms to identify publications that, besides polymeric sampling data, addressed either fish, shellfish, lipid-normalization, bioconcentration, bioaccumulation, biomagnification, biouptake, tropic transfer, food chain, or food web. A full list of the search terms, as entered into Web of Science, is provided in Table S1.

A total of 349 different publications were retrieved in the literature search. Of these, 43 publications addressed polymeric sampling and bioaccumulation by fish and/or shellfish. The 43 publications were carefully reviewed, and corresponding $\mathrm{PCB}$ equilibrium polymer concentrations and lipid-normalized $\mathrm{PCB}$ concentrations in fish and shellfish were retrieved from a total of 10 publications. ${ }^{4,5,11-18}$ The data from these publications formed the data set for the data analysis together with data collected from three additional publications, ${ }^{19-21}$ selected from the reference lists in the 43 publications identified in the literature search. In this way, data from a total of 13 peer-reviewed scientific publications formed the data set for this review. An overview of these publications and selected experimental details are provided in Table S2. In several cases, authors of the publications generously provided raw data in addition to general clarifications to the relevant studies. Al assistance from these authors is greatly appreciated and deeply acknowledged.

\begin{tabular}{|c|c|c|}
\hline Primary search term & Secondary search term & Hits (papers) \\
\hline \multirow[t]{11}{*}{ "passive sampl*" } & fish & 134 \\
\hline & shellfish & 34 \\
\hline & "lipid normali*” & 16 \\
\hline & bioconcentrat* & 47 \\
\hline & bioaccumulat* & 170 \\
\hline & biomagnif* & 8 \\
\hline & biouptake & 2 \\
\hline & "bio uptake" & 1 \\
\hline & "trophic transfer" & 4 \\
\hline & "food chain" & 8 \\
\hline & "food web" & 15 \\
\hline \multirow[t]{11}{*}{ "equilibrium sampl*" } & fish & 13 \\
\hline & shellfish & 0 \\
\hline & "lipid normali*” & 9 \\
\hline & bioconcentrat* & 4 \\
\hline & bioaccumulat* & 14 \\
\hline & biomagnif* & 2 \\
\hline & biouptake & 1 \\
\hline & "bio uptake" & 0 \\
\hline & "trophic transfer" & 0 \\
\hline & "food chain" & 1 \\
\hline & "food web" & 0 \\
\hline \multirow[t]{11}{*}{ "polymer* sampl*” } & fish & 1 \\
\hline & shellfish & 0 \\
\hline & "lipid normali*" & 0 \\
\hline & bioconcentrat* & 0 \\
\hline & bioaccumulat* & 2 \\
\hline & biomagnif* & 1 \\
\hline & biouptake & 0 \\
\hline & "bio uptake" & 0 \\
\hline & "trophic transfer" & 0 \\
\hline & "food chain" & 0 \\
\hline & "food web" & 1 \\
\hline
\end{tabular}




\begin{tabular}{|c|c|c|c|c|c|c|c|}
\hline Reference & Location & Medium & & Polymeric s & ipling & Bioaccumula & \\
\hline & & & Type & Exposure & Equilibrium & Name & Exposure \\
\hline $\begin{array}{l}\text { Allan et al, } \\
2013^{11}\end{array}$ & $\begin{array}{l}\text { River Alna, } \\
\text { Norway }\end{array}$ & Water column & AlteSil strips & $\begin{array}{l}\text { In situ, } \\
28 \text { days }\end{array}$ & Inferred via PRCs & Brown trout (Salmo trutta) & $\begin{array}{l}\text { In situ (caged), } \\
28 \text { days }\end{array}$ \\
\hline $\begin{array}{l}\text { Echols et al, } \\
2000^{19}\end{array}$ & $\begin{array}{l}\text { Saginaw River, } \\
\text { MI, USA }\end{array}$ & Water column & SPMDs & $\begin{array}{l}\text { In situ, } \\
28 \text { days }\end{array}$ & Assumed & Channel catfish (Ictalurus punctatus) & $\begin{array}{l}\text { In situ (caged), } \\
28 \text { days }\end{array}$ \\
\hline $\begin{array}{l}\text { Fadaei et al, } \\
2015^{12 *}\end{array}$ & $\begin{array}{l}\text { Grasse River, } \\
\text { NY, USA }\end{array}$ & $\begin{array}{l}\text { Sediment interstitial } \\
\text { water }\end{array}$ & POM strips & $\begin{array}{l}\text { Ex situ, } \\
90 \text { days }\end{array}$ & $\begin{array}{l}\text { Confirmed via time } \\
\text { series }\end{array}$ & Zebrafish (Danio rerio) & $\begin{array}{l}\text { Ex situ, } \\
90 \text { days }\end{array}$ \\
\hline & $\begin{array}{l}\text { (artificial sea } \\
\text { water) }\end{array}$ & Water column & POM strips & $\begin{array}{l}\text { Ex situ, } \\
90 \text { days }\end{array}$ & Assumed & Zebrafish (Danio rerio) & $\begin{array}{l}\text { Ex situ, } \\
90 \text { days }\end{array}$ \\
\hline $\begin{array}{l}\text { Gale et al, } \\
1997^{13}\end{array}$ & $\begin{array}{l}\text { Saginaw River, } \\
\text { MI, USA }\end{array}$ & Water column & SPMDs & $\begin{array}{l}\text { In situ, } \\
28 \text { days }\end{array}$ & Assumed & Channel catfish (Ictalurus punctatus) & $\begin{array}{l}\text { In situ (caged), } \\
28 \text { days }\end{array}$ \\
\hline $\begin{array}{l}\text { Jahnke et al, } \\
2012^{20}\end{array}$ & $\begin{array}{l}\text { Baltic Sea, } \\
\text { Sweden }\end{array}$ & $\begin{array}{l}\text { Sediment interstitial } \\
\text { water }\end{array}$ & $\begin{array}{l}\text { PDMS coated } \\
\text { glass jars }\end{array}$ & $\begin{array}{l}\text { Ex situ, } \\
2 \text { weeks }\end{array}$ & $\begin{array}{l}\text { Confirmed via } \\
\text { multiple thicknesses }\end{array}$ & Herring (Clupea harengus) & $\begin{array}{l}\text { Indigenous, } \\
\text { wild caught }\end{array}$ \\
\hline $\begin{array}{l}\text { Jahnke et al, } \\
2014^{4}\end{array}$ & $\begin{array}{l}\text { Lake Ången, } \\
\text { Sweden }\end{array}$ & $\begin{array}{l}\text { Sediment interstitial } \\
\text { water }\end{array}$ & $\begin{array}{l}\text { DC coated glass } \\
\text { jars }\end{array}$ & $\begin{array}{l}\text { Ex situ, } \\
2 \text { weeks }\end{array}$ & $\begin{array}{l}\text { Confirmed via } \\
\text { multiple thicknesses }\end{array}$ & European eel (Anguilla anguilla) & $\begin{array}{l}\text { Indigenous, } \\
\text { wild caught }\end{array}$ \\
\hline $\begin{array}{l}\text { Jahnke et al, } \\
2014^{5}\end{array}$ & $\begin{array}{l}\text { Lake Ången, } \\
\text { Sweden }\end{array}$ & $\begin{array}{l}\text { Sediment interstitial } \\
\text { water }\end{array}$ & $\begin{array}{l}\text { DC coated glass } \\
\text { jars }\end{array}$ & $\begin{array}{l}\text { Ex situ, } \\
2 \text { weeks }\end{array}$ & $\begin{array}{l}\text { Confirmed via } \\
\text { multiple thicknesses }\end{array}$ & $\begin{array}{l}\text { European eel (Anguilla anguilla) } \\
\text { Perch (Perca fluviatilis) } \\
\text { Pike (Esox lucius) } \\
\text { Pikeperch (Sander lucioperca) } \\
\text { Roach (Rutilus rutilus) }\end{array}$ & $\begin{array}{l}\text { Indigenous, } \\
\text { wild caught }\end{array}$ \\
\hline
\end{tabular}




\section{Table S2. Continued.}

Reference

Location

Medium

Polymeric sampling

\begin{tabular}{|c|c|c|c|c|c|c|c|}
\hline & & & Type & Exposure & Equilibrium & Name & Exposure \\
\hline \multirow[t]{2}{*}{$\begin{array}{l}\text { Khairy et al, } \\
2014^{14}\end{array}$} & $\begin{array}{l}\text { Passaic River, } \\
\text { NJ, USA }\end{array}$ & $\begin{array}{l}\text { Sediment interstitial } \\
\text { water }\end{array}$ & LDPE sheets & $\begin{array}{l}\text { Ex situ, } \\
8 \text { weeks }\end{array}$ & Inferred via PRCs & $\begin{array}{l}\text { American eel (Anguilla rostrata) } \\
\text { Atlantic silverside (Menidia menidia) } \\
\text { Banded killifish (Fundulus diaphanus) } \\
\text { Blue crab (Callinectes sapidus) } \\
\text { Gizzard shad (Dorosoma cepedianum) } \\
\text { Mummichog (Fundulus heteroclitus) } \\
\text { Pumpkinseed sunfish (Lepomis gibbosus) } \\
\text { Silvery minnow (Hybognathus regius) } \\
\text { Striped bass (Morone saxatilis) } \\
\text { White perch (Morone americana) }\end{array}$ & $\begin{array}{l}\text { Indigenous, } \\
\text { wild caught }\end{array}$ \\
\hline & $\begin{array}{l}\text { Passaic River, } \\
\text { NJ, USA }\end{array}$ & Water column & LDPE sheets & $\begin{array}{l}\text { In situ, } \\
\sim 2 \text { months }\end{array}$ & Inferred via PRCs & $\begin{array}{l}\text { American eel (Anguilla rostrata) } \\
\text { Atlantic silverside (Menidia menidia) } \\
\text { Banded killifish (Fundulus diaphanus) } \\
\text { Blue crab (Callinectes sapidus) } \\
\text { Gizzard shad (Dorosoma cepedianum) } \\
\text { Mummichog (Fundulus heteroclitus) } \\
\text { Pumpkinseed sunfish (Lepomis gibbosus) } \\
\text { Silvery minnow (Hybognathus regius) } \\
\text { Striped bass (Morone saxatilis) } \\
\text { White perch (Morone americana) }\end{array}$ & $\begin{array}{l}\text { Indigenous, } \\
\text { wild caught }\end{array}$ \\
\hline $\begin{array}{l}\text { Morgan \& Lohmann, } \\
2010^{15}\end{array}$ & $\begin{array}{l}\text { Narragansett Bay, } \\
\text { RI, USA }\end{array}$ & $\begin{array}{l}\text { Sediment interstitial } \\
\text { water }\end{array}$ & LDPE strips & $\begin{array}{l}\text { Ex situ, } \\
3 \text { weeks }\end{array}$ & Assumed & $\begin{array}{l}\text { American butterfish (Peprilus triacanthus) } \\
\text { American lobster (Homarus americanus) } \\
\text { Crab (Cancer spp.) } \\
\text { Long-finned squid (Loligo pealei) } \\
\text { Scup (Stenotomus chrysops) }\end{array}$ & $\begin{array}{l}\text { Indigenous, } \\
\text { wild caught }\end{array}$ \\
\hline $\begin{array}{l}\text { Schäfer et al, } \\
2015^{16}\end{array}$ & $\begin{array}{l}\text { River Elbe, } \\
\text { Germany }\end{array}$ & $\begin{array}{l}\text { Sediment interstitial } \\
\text { water }\end{array}$ & $\begin{array}{l}\text { DC coated } \\
\text { glass jars }\end{array}$ & $\begin{array}{l}\text { Ex situ, } \\
2 \text { weeks }\end{array}$ & $\begin{array}{l}\text { Confirmed via } \\
\text { multiple thicknesses }\end{array}$ & Common bream (Abramis brama) & $\begin{array}{l}\text { Indigenous, } \\
\text { wild caught }\end{array}$ \\
\hline
\end{tabular}




\section{Table S2. Continued.}

Reference

Location

Medium

Polymeric sampling

Exposure Equilibrium

Name

Bioaccumulation

\begin{tabular}{|c|c|c|c|c|c|c|c|}
\hline $\begin{array}{l}\text { Sobek et al, } \\
2014^{17}\end{array}$ & $\begin{array}{l}\text { Baltic Sea, } \\
\text { Sweden }\end{array}$ & $\begin{array}{l}\text { Sediment interstitial } \\
\text { water }\end{array}$ & POM strips & $\begin{array}{l}\text { Ex situ, } \\
120 \text { days }\end{array}$ & Assumed & Perch (Perca fluviatilis) & $\begin{array}{l}\text { Indigenous, } \\
\text { wild caught }\end{array}$ \\
\hline & $\begin{array}{l}\text { Baltic Sea, } \\
\text { Sweden }\end{array}$ & Water column & POM strips & $\begin{array}{l}\text { In situ, } \\
>120 \text { days }\end{array}$ & Assumed & Perch (Perca fluviatilis) & $\begin{array}{l}\text { Indigenous, } \\
\text { wild caught }\end{array}$ \\
\hline $\begin{array}{l}\text { Verweij et al, } \\
2004^{18}\end{array}$ & $\begin{array}{l}\text { Nine locations, } \\
\text { The Netherlands }\end{array}$ & Water column & SPMDs & $\begin{array}{l}\text { In situ, } \\
4 \text { weeks }\end{array}$ & Inferred via PRCs & Carp (Cyprinus carpio) & $\begin{array}{l}\text { In situ (caged), } \\
4 \text { weeks }\end{array}$ \\
\hline $\begin{array}{l}\text { Wang et al, } \\
2002^{21}\end{array}$ & $\begin{array}{l}\text { Huaihe River, } \\
\text { China }\end{array}$ & Water column & SPMDs & $\begin{array}{l}\text { In situ, } \\
28 \text { days }\end{array}$ & Assumed & Grass carp (Cyprinus carpio) & $\begin{array}{l}\text { Indigenous, } \\
\text { wild caught }\end{array}$ \\
\hline
\end{tabular}

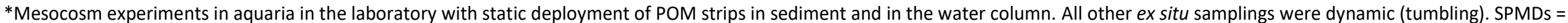
semipermeable membrane devices; POM = polyoxymethylene; PDMS = polydimethylsiloxane; DC = Dow Corning 1-2577 conformal coating; LDPE = low density polyethylene. PRCs =

performance reference compounds. 


\begin{tabular}{|c|c|c|c|c|c|}
\hline Polymer type & Medium & Organism & $\begin{array}{l}\text { Total number } \\
\text { of regressions }\end{array}$ & $\begin{array}{l}\text { Total number } \\
\text { of data points }\end{array}$ & Reference(s) \\
\hline AlteSil & Water column & Fish & 8 & 40 & Allan et al, 2013 \\
\hline DC & Sediment interstitial water & Fish & 13 & 87 & Jahnke et al, 2014a; Jahnke et al, 2014b; Schäfer et al, 2015 \\
\hline \multirow[t]{4}{*}{ LDPE } & Sediment interstitial water & Fish & 45 & 692 & Khairy et al, 2014; Morgan and Lohmann, 2010 \\
\hline & Sediment interstitial water & Shellfish & 30 & 278 & Khairy et al, 2014; Morgan and Lohmann, 2010 \\
\hline & Water column & Fish & 16 & 464 & Khairy et al, 2014 \\
\hline & Water column & Shellfish & 1 & 29 & Khairy et al, 2014 \\
\hline PDMS & Sediment interstitial water & Fish & 1 & 7 & Jahnke et al, 2012 \\
\hline \multirow[t]{2}{*}{ POM } & Sediment interstitial water & Fish & 5 & 95 & Fadaei et al, 2015; Sobek et al, 2014 \\
\hline & Water column & Fish & 4 & 123 & Fadaei et al, 2015; Sobek et al, 2014 \\
\hline SPMD & Water column & Fish & 21 & 172 & Echols et al, 2000; Gale et al, 1997; Verweij et al, 2004; Wang et al, 2002 \\
\hline
\end{tabular}


Table S4. Summary of the bioaccumulation data across the 13 peer-reviewed scientific publications.

\begin{tabular}{|c|c|c|c|c|c|c|c|}
\hline Organism & Common name & Scientific name & Migration* & Tropic level* & $\begin{array}{l}\text { Total number } \\
\text { of regressions }\end{array}$ & $\begin{array}{l}\text { Total number } \\
\text { of data points }\end{array}$ & References \\
\hline \multirow[t]{22}{*}{ Fish } & Common bream & Abramis brama & Potamodromous & $3.1 \pm 0.1$ & 3 & 21 & Schäfer et al, 2015 \\
\hline & European eel If & Anguilla anguilla & Catadromous & $3.6 \pm 0.3$ & 6 & 38 & Jahnke et al, 2014a; Jahnke et al, 2014b \\
\hline & American eel If & Anguilla rostrata & Catadromous & $3.8 \pm 0.2$ & 4 & 116 & Khairy et al, 2014 \\
\hline & Herring II & Clupea harengus & Oceanodromous & $3.4 \pm 0.1$ & 1 & 7 & Jahnke et al, 2012 \\
\hline & Carp If & Cyprinus carpio & Potamodromous & $3.1 \pm 0.0$ & 11 & 76 & Verweij et al, 2004; Wang et al, 2002 \\
\hline & Zebrafish & Danio rerio & Nonmigratory & $3.1 \pm 0.1$ & 2 & 157 & Fadaei et al, 2015 \\
\hline & Gizzard shad & Dorosoma cepedianum & Anadromous & $2.4 \pm 0.21$ & 2 & 58 & Khairy et al, 2014 \\
\hline & Pike II & Esox lucius & Potamodromous & $4.1 \pm 0.4$ & 1 & 7 & Jahnke et al, 2014b \\
\hline & Banded killifish & Fundulus diaphanus & Nonmigratory & $3.3 \pm 0.2$ & 2 & 58 & Khairy et al, 2014 \\
\hline & Mummichog & Fundulus heteroclitus & Nonmigratory & $3.6 \pm 0.48$ & 2 & 58 & Khairy et al, 2014 \\
\hline & Silvery minnow & Hybognathus regius & Nonmigratory & $2.0 \pm 0.00$ & 4 & 116 & Khairy et al, 2014 \\
\hline & Channel catfish II & Ictalurus punctatus & Nonmigratory & $4.2 \pm 0.3$ & 10 & 96 & Echols et al, 2000; Gale et al, 1997 \\
\hline & Pumpkinseed sunfish & Lepomis gibbosus & Potamodromous & $3.3 \pm 0.1$ & 2 & 58 & Khairy et al, 2014 \\
\hline & Atlantic silverside & Menidia menidia & Oceanodromous & $3.2 \pm 0.1$ & 4 & 116 & Khairy et al, 2014 \\
\hline & White perch If & Morone americana & Anadromous & $3.1 \pm 0.35$ & 4 & 116 & Khairy et al, 2014 \\
\hline & Striped bass If & Morone saxatilis & Anadromous & $4.7 \pm 0.2$ & 8 & 232 & Khairy et al, 2014 \\
\hline & American butterfish II & Peprilus triacanthus & Oceanodromous & $3.9 \pm 0.0$ & 11 & 86 & Morgan and Lohmann, 2010 \\
\hline & Perch If & Perca fluviatilis & Anadromous & $4.4 \pm 0.0$ & 8 & 68 & Jahnke et al, 2014b; Sobek et al, 2014 \\
\hline & Roach & Rutilus rutilus & Potamodromous & $3.0 \pm 0.0$ & 1 & 7 & Jahnke et al, 2014b \\
\hline & Brown trout Il & Salmo trutta & Anadromous & $3.4 \pm 0.1$ & 8 & 40 & Allan et al, 2013 \\
\hline & Pikeperch II & Sander lucioperca & Potamodromous & $4.0 \pm 0.78$ & 1 & 7 & Jahnke et al, 2014b \\
\hline & Scup II & Stenotomus chrysops & Oceanodromous & $3.8 \pm 0.1$ & 18 & 142 & Morgan and Lohmann, 2010 \\
\hline \multirow[t]{4}{*}{ Shellfish } & Blue crab If & Callinectes sapidus & - & - & 2 & 58 & Khairy et al, 2014 \\
\hline & Crab & Cancer spp. & - & - & 4 & 36 & Morgan and Lohmann, 2010 \\
\hline & American lobster II & Homarus americanus & - & - & 12 & 102 & Morgan and Lohmann, 2010 \\
\hline & Long-finned squid II & Loligo pealei & - & - & 13 & 111 & Morgan and Lohmann, 2010 \\
\hline
\end{tabular}

Il Fish and shellfish routinely consumed by humans. *Data from www.fishbase.org (accessed on August 11, 2019). 
Figure S2. Lipid-normalized concentrations in fish/shellfish (y axis, $\log C_{L}, n g^{-1}$ lipid) linked to equilibrium polymer concentrations ( $x$ axis, log $C_{p}$, ng $g^{-1}$ polymer) by linear regression (see Table S5). Each individual data point represents data for a different PCB congener, and the gray area visualizes the hypothetical 1:1 relationship \pm a factor of 10 .
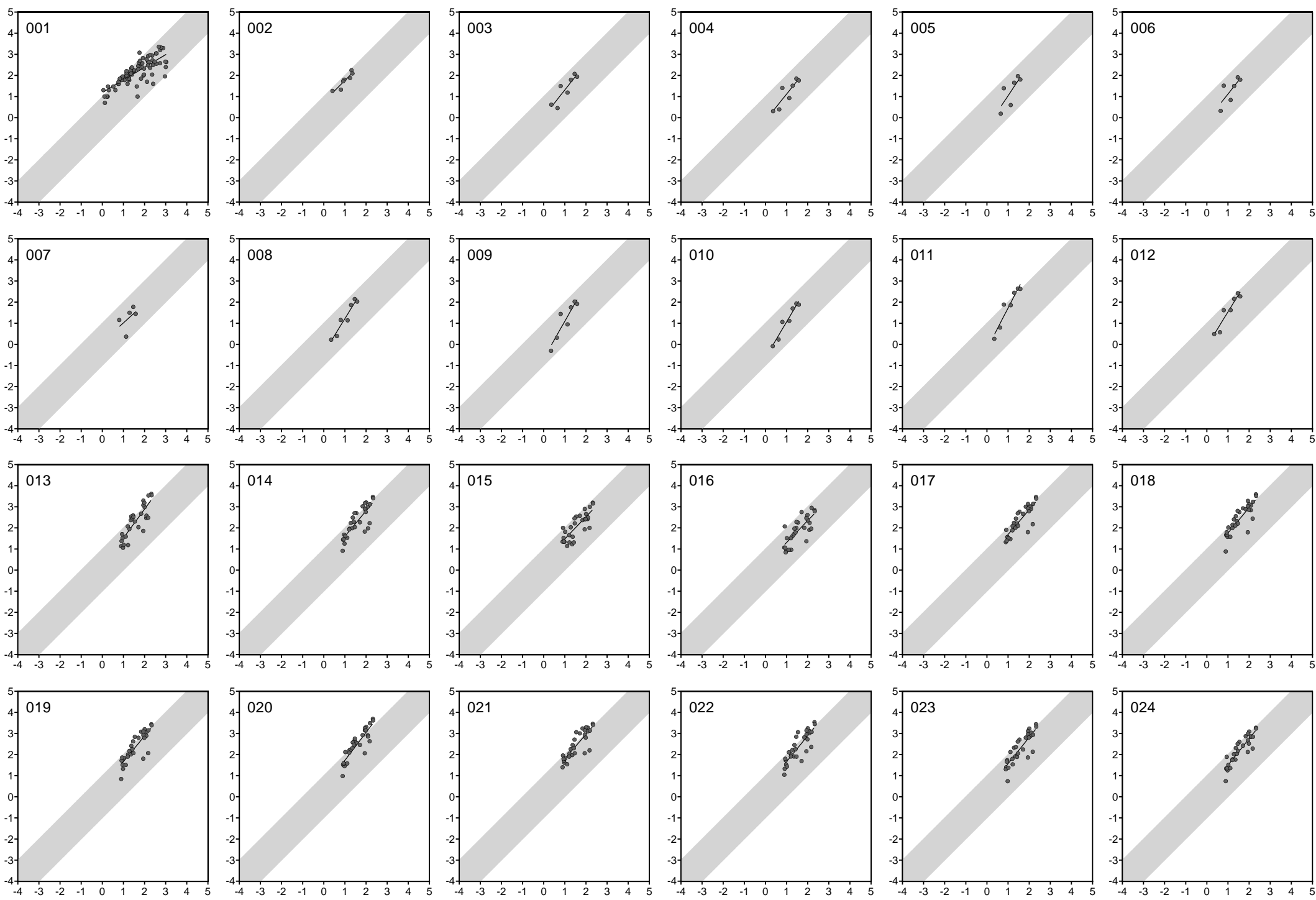
Figure S2. Continued.
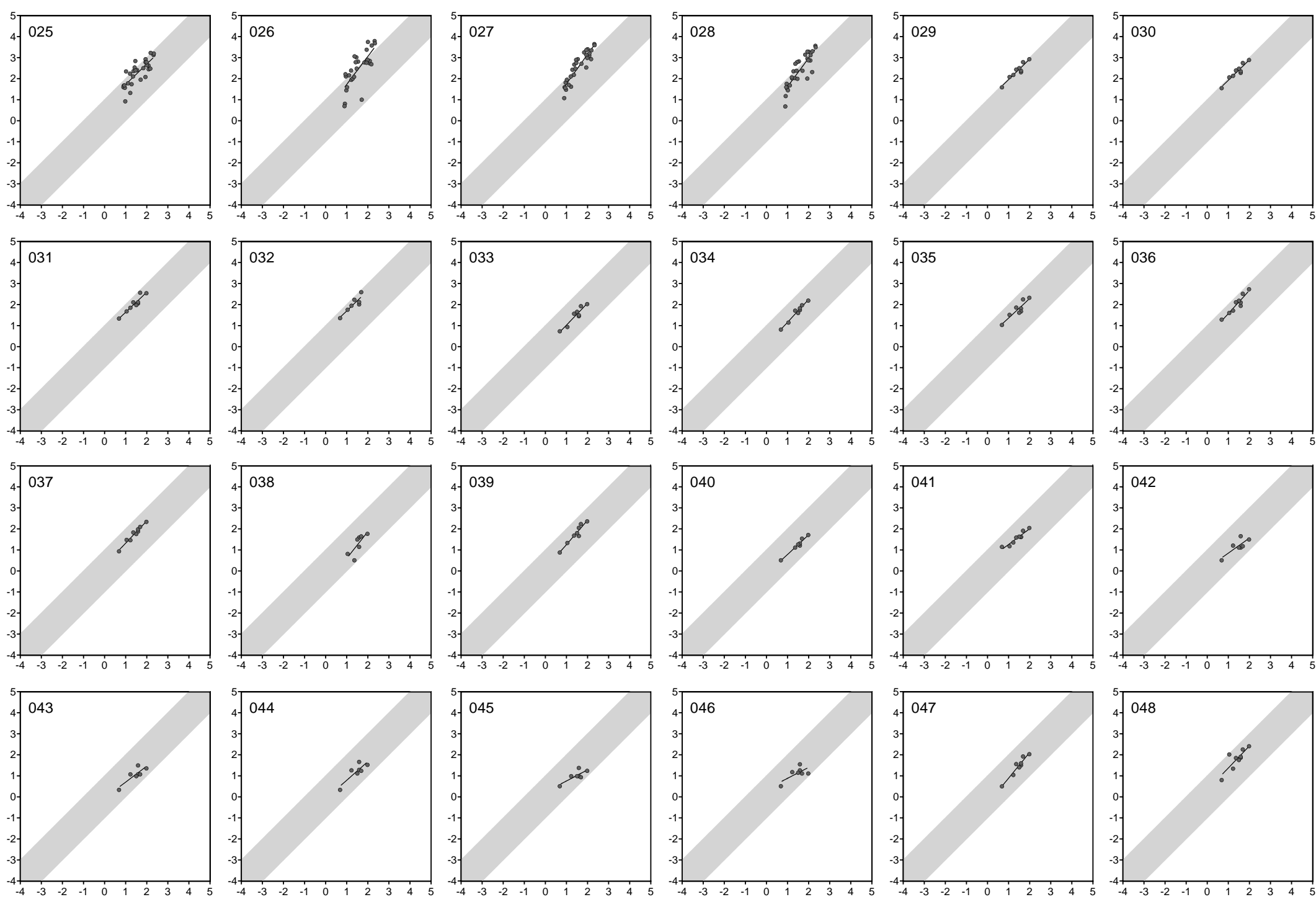


\section{Figure S2. Continued.}
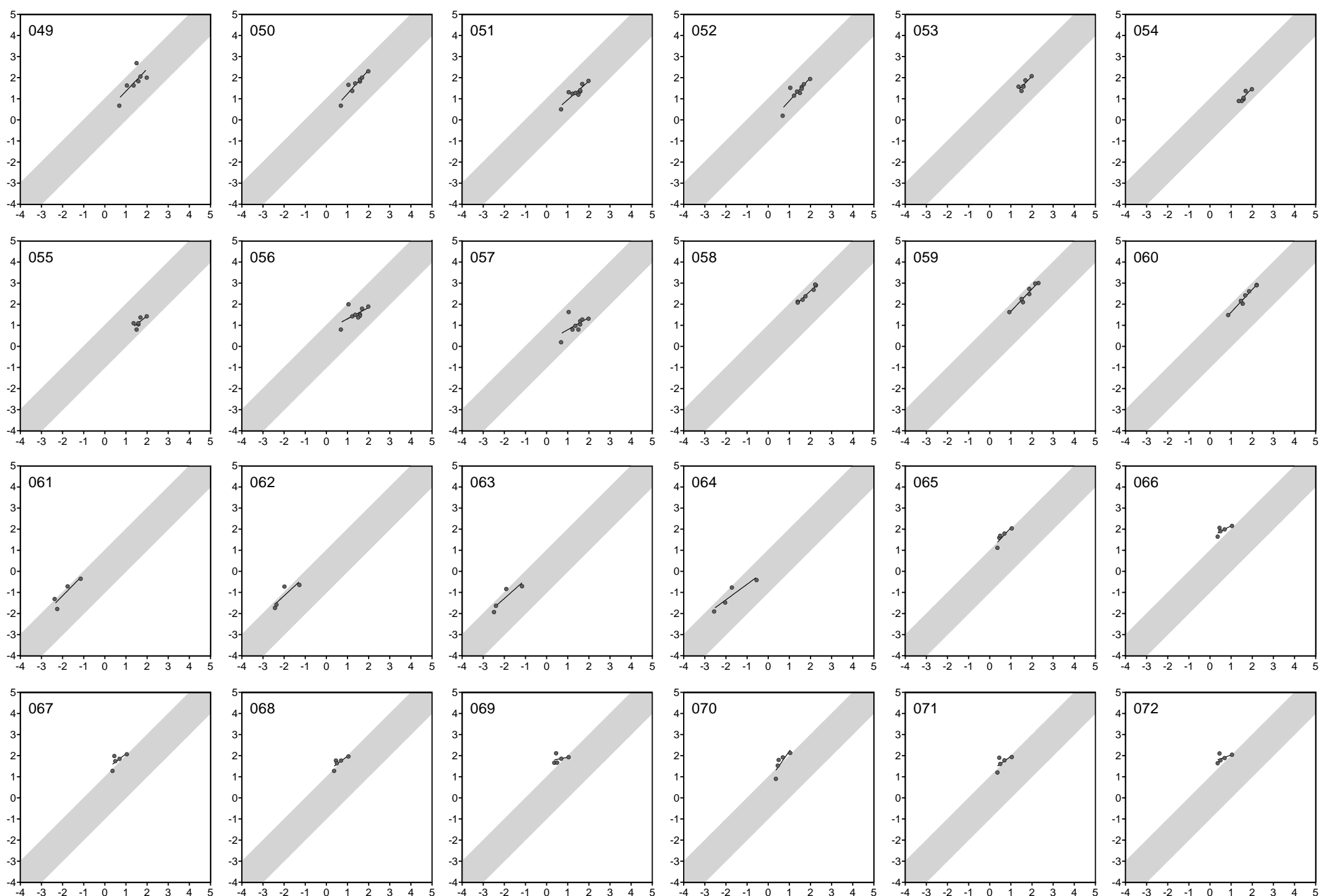

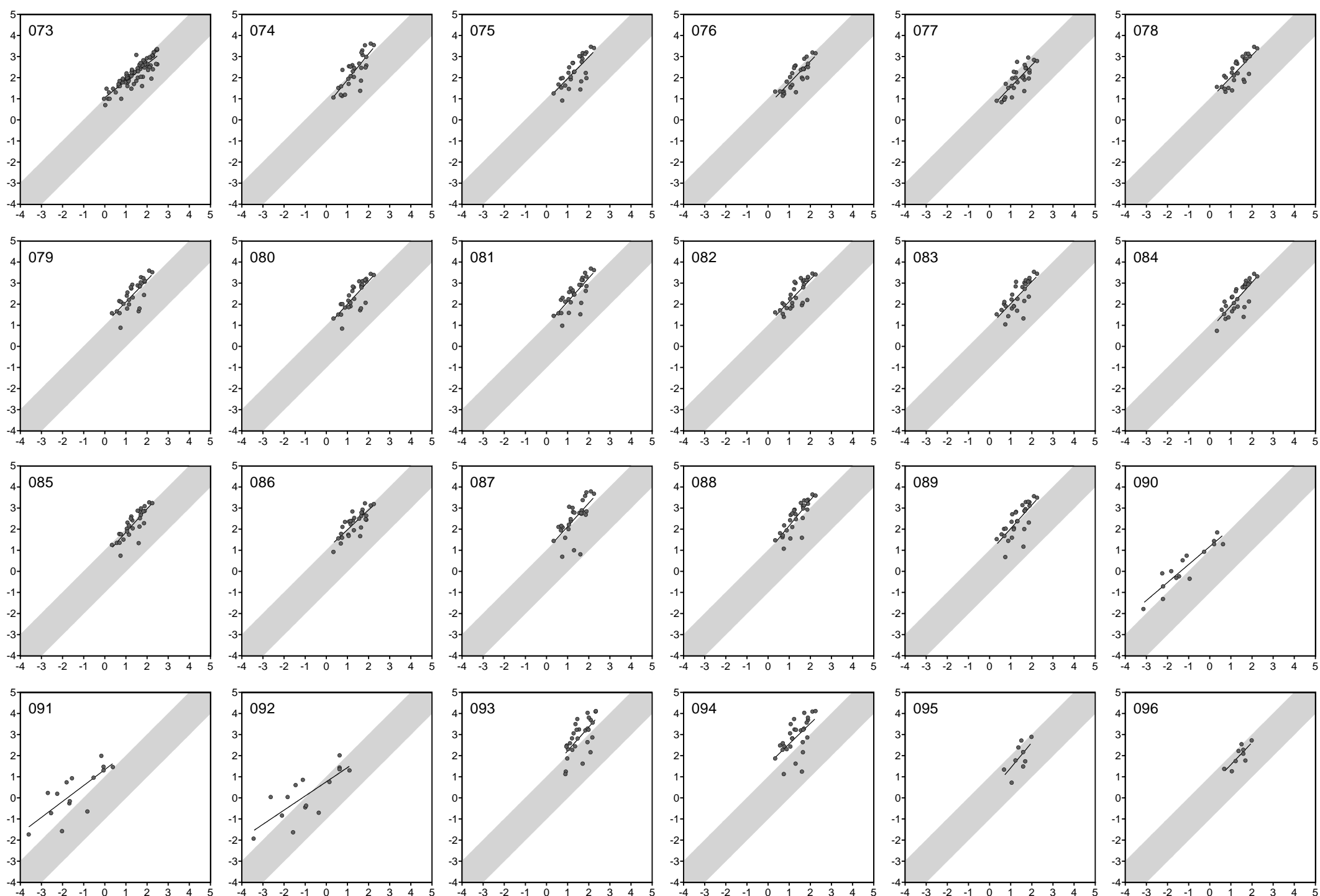


\section{Figure S2. Continued.}
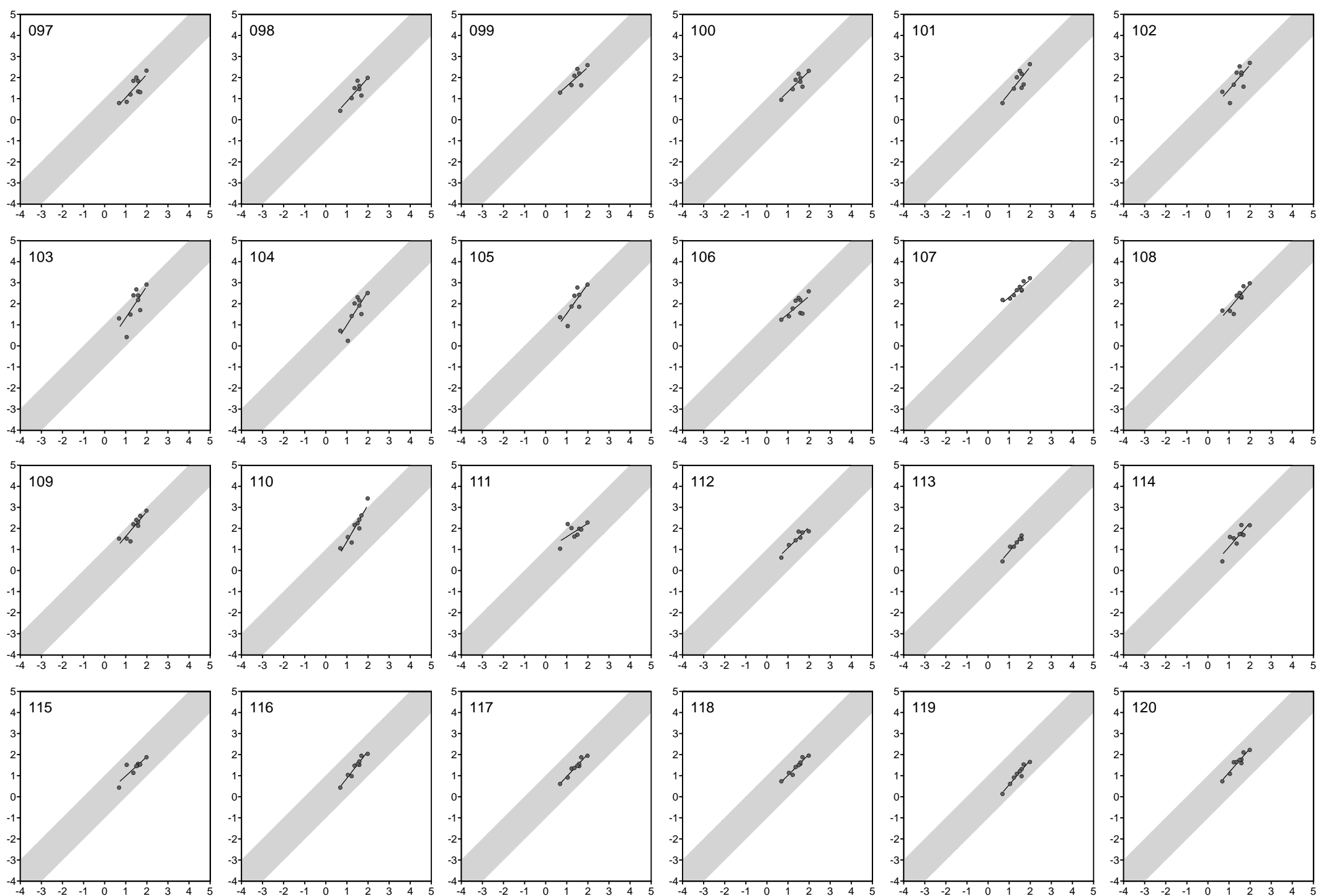

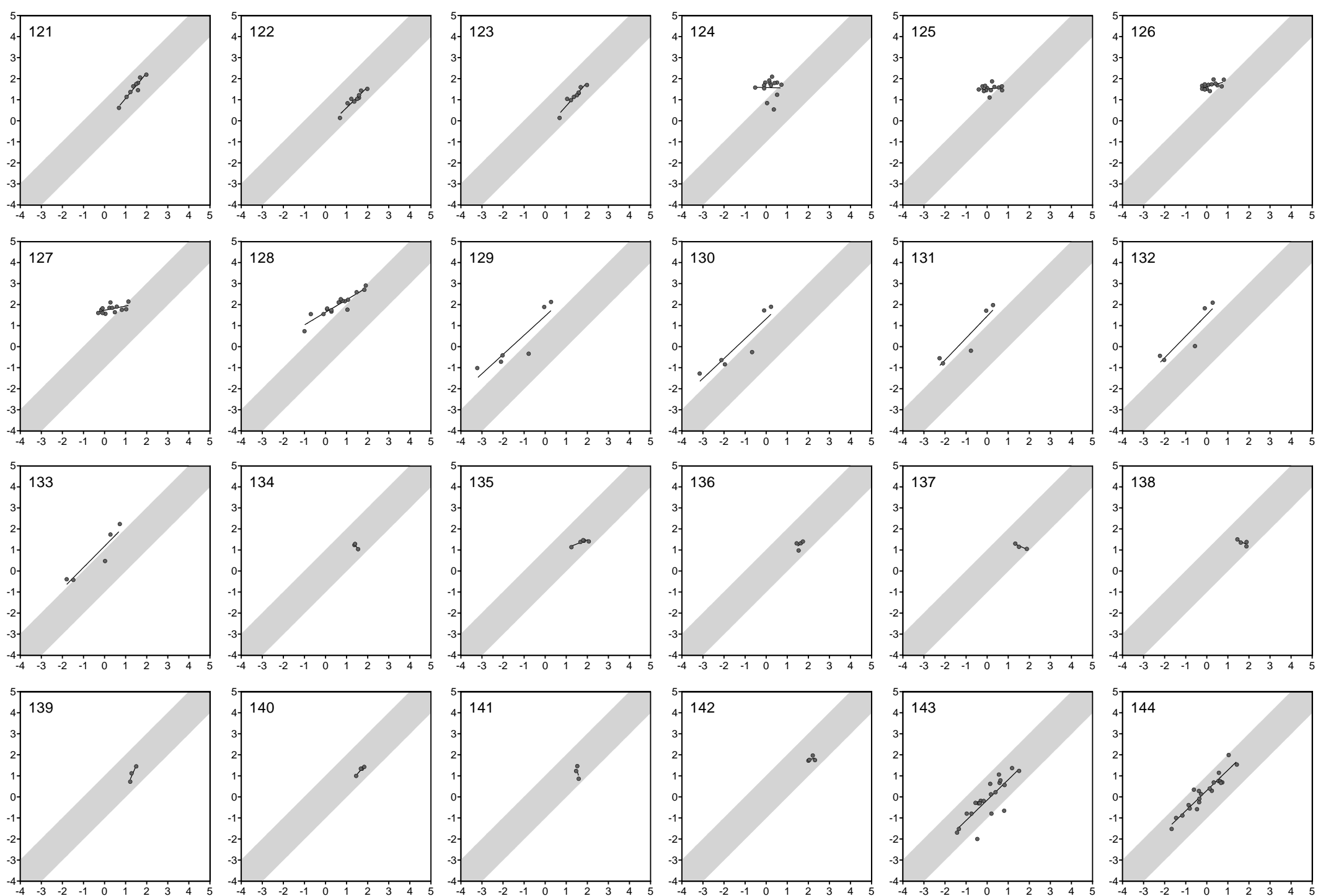


\begin{tabular}{|c|c|c|c|c|c|c|c|c|c|}
\hline Panel & Fish species & Polymer & Slope & Intercept & $p$-value & $r^{2}$ & Sy.x & $\mathrm{n}$ & Reference \\
\hline 001 & Danio rerio & POM & 0.60 & 1.19 & $<0.0001$ & 0.62 & 0.37 & 79 & Fadaei et al. (2015) \\
\hline 002 & Clupea harengus & PDMS* & 1.00 & 0.76 & 0.0035 & 0.84 & 0.16 & 7 & Jahnke et al. (2012) \\
\hline 003 & Anguilla anguilla & $\mathrm{DC}^{*}$ & 1.26 & 0.05 & 0.0076 & 0.79 & 0.32 & 7 & Jahnke et al. (2014a) \\
\hline 004 & Anguilla anguilla & $\mathrm{DC}^{*}$ & 1.24 & -0.13 & 0.0085 & 0.78 & 0.33 & 7 & Jahnke et al. (2014a) \\
\hline 005 & Anguilla anguilla & $\mathrm{DC}^{*}$ & 1.50 & -0.46 & 0.0722 & 0.60 & 0.51 & 6 & Jahnke et al. (2014a) \\
\hline 006 & Anguilla anguilla & $\mathrm{DC}^{*}$ & 1.27 & -0.15 & 0.0799 & 0.58 & 0.45 & 6 & Jahnke et al. (2014a) \\
\hline 007 & Anguilla anguilla & $\mathrm{DC}^{*}$ & 0.91 & 0.11 & 0.3754 & 0.26 & 0.53 & 5 & Jahnke et al. (2014a) \\
\hline 008 & Rutilus rutilus & $\mathrm{DC}^{*}$ & 1.63 & -0.41 & 0.0006 & 0.92 & 0.23 & 7 & Jahnke et al. (2014b) \\
\hline 009 & Anguilla anguilla & $\mathrm{DC}^{*}$ & 1.76 & -0.67 & 0.0041 & 0.83 & 0.40 & 7 & Jahnke et al. (2014b) \\
\hline 010 & Sander lucioperca & $\mathrm{DC}^{*}$ & 1.69 & -0.63 & 0.0003 & 0.94 & 0.22 & 7 & Jahnke et al. (2014b) \\
\hline 011 & Perca fluviatilis & $\mathrm{DC}^{*}$ & 1.94 & -0.22 & 0.0009 & 0.91 & 0.31 & 7 & Jahnke et al. (2014b) \\
\hline 012 & Esox lucius & $\mathrm{DC}^{*}$ & 1.63 & -0.09 & 0.0012 & 0.90 & 0.28 & 7 & Jahnke et al. (2014b) \\
\hline 013 & Morone saxatilis & LDPE & 1.37 & 0.15 & $<0.0001$ & 0.73 & 0.41 & 29 & Khairy et al. (2014) \\
\hline 014 & Hybognathus regius & LDPE & 1.20 & 0.40 & $<0.0001$ & 0.68 & 0.40 & 29 & Khairy et al. (2014) \\
\hline 015 & Dorosoma cepedianum & LDPE & 1.05 & 0.43 & $<0.0001$ & 0.65 & 0.37 & 29 & Khairy et al. (2014) \\
\hline 016 & Fundulus heteroclitus & LDPE & 1.04 & 0.27 & $<0.0001$ & 0.60 & 0.41 & 29 & Khairy et al. (2014) \\
\hline 017 & Menidia menidia & LDPE & 1.17 & 0.52 & $<0.0001$ & 0.74 & 0.34 & 29 & Khairy et al. (2014) \\
\hline 018 & Menidia menidia & LDPE & 1.20 & 0.56 & $<0.0001$ & 0.72 & 0.36 & 29 & Khairy et al. (2014) \\
\hline 019 & Morone americana & LDPE & 1.21 & 0.46 & $<0.0001$ & 0.70 & 0.38 & 29 & Khairy et al. (2014) \\
\hline 020 & Morone saxatilis & LDPE & 1.32 & 0.41 & $<0.0001$ & 0.79 & 0.33 & 29 & Khairy et al. (2014) \\
\hline 021 & Morone americana & LDPE & 1.16 & 0.64 & $<0.0001$ & 0.72 & 0.35 & 29 & Khairy et al. (2014) \\
\hline 022 & Lepomis gibbosus & LDPE & 1.22 & 0.44 & $<0.0001$ & 0.71 & 0.38 & 29 & Khairy et al. (2014) \\
\hline 023 & Hybognathus regius & LDPE & 1.25 & 0.32 & $<0.0001$ & 0.71 & 0.38 & 29 & Khairy et al. (2014) \\
\hline 024 & Fundulus diaphanus & LDPE & 1.26 & 0.24 & $<0.0001$ & 0.80 & 0.30 & 29 & Khairy et al. (2014) \\
\hline 025 & Anguilla rostrata & LDPE & 0.97 & 0.75 & $<0.0001$ & 0.64 & 0.35 & 29 & Khairy et al. (2014) \\
\hline 026 & Anguilla rostrata & LDPE & 1.33 & 0.40 & $<0.0001$ & 0.57 & 0.55 & 29 & Khairy et al. (2014) \\
\hline 027 & Morone saxatilis & LDPE & 1.40 & 0.36 & $<0.0001$ & 0.85 & 0.29 & 29 & Khairy et al. (2014) \\
\hline 028 & Morone saxatilis & LDPE & 1.35 & 0.27 & $<0.0001$ & 0.74 & 0.39 & 29 & Khairy et al. (2014) \\
\hline 029 & Stenotomus chrysops & LDPE & 0.97 & 0.98 & 0.0001 & 0.90 & 0.14 & 9 & Morgan and Lohmann (2010) \\
\hline 030 & Stenotomus chrysops & LDPE & 0.97 & 0.95 & $<0.0001$ & 0.90 & 0.13 & 9 & Morgan and Lohmann (2010) \\
\hline 031 & Stenotomus chrysops & LDPE & 0.95 & 0.68 & 0.0002 & 0.88 & 0.14 & 9 & Morgan and Lohmann (2010) \\
\hline 032 & Stenotomus chrysops & LDPE & 0.97 & 0.73 & 0.0062 & 0.80 & 0.19 & 7 & Morgan and Lohmann (2010) \\
\hline
\end{tabular}




\section{Table S5. Continued.}

\begin{tabular}{|c|c|c|c|c|c|c|c|c|c|}
\hline Panel & Fish species & Polymer & Slope & Intercept & p-value & $r^{2}$ & Sy.x & $\mathrm{n}$ & Reference \\
\hline 033 & Stenotomus chrysops & LDPE & 1.04 & -0.02 & 0.0004 & 0.89 & 0.16 & 8 & Morgan and Lohmann (2010) \\
\hline 034 & Stenotomus chrysops & LDPE & 1.09 & 0.07 & $<0.0001$ & 0.96 & 0.09 & 8 & Morgan and Lohmann (2010) \\
\hline 035 & Stenotomus chrysops & LDPE & 0.93 & 0.43 & 0.0019 & 0.82 & 0.19 & 8 & Morgan and Lohmann (2010) \\
\hline 036 & Stenotomus chrysops & LDPE & 1.10 & 0.47 & 0.0001 & 0.89 & 0.16 & 9 & Morgan and Lohmann (2010) \\
\hline 037 & Stenotomus chrysops & LDPE & 1.05 & 0.27 & $<0.0001$ & 0.96 & 0.09 & 9 & Morgan and Lohmann (2010) \\
\hline 038 & Stenotomus chrysops & LDPE & 1.27 & -0.68 & 0.0393 & 0.61 & 0.33 & 7 & Morgan and Lohmann (2010) \\
\hline 039 & Stenotomus chrysops & LDPE & 1.16 & 0.10 & 0.0001 & 0.93 & 0.14 & 8 & Morgan and Lohmann (2010) \\
\hline 040 & Stenotomus chrysops & LDPE & 0.93 & -0.15 & $<0.0001$ & 0.97 & 0.08 & 7 & Morgan and Lohmann (2010) \\
\hline 041 & Stenotomus chrysops & LDPE & 0.75 & 0.51 & $<0.0001$ & 0.90 & 0.10 & 9 & Morgan and Lohmann (2010) \\
\hline 042 & Stenotomus chrysops & LDPE & 0.71 & 0.13 & 0.026 & 0.66 & 0.23 & 7 & Morgan and Lohmann (2010) \\
\hline 043 & Stenotomus chrysops & LDPE & 0.76 & -0.06 & 0.0152 & 0.72 & 0.21 & 7 & Morgan and Lohmann (2010) \\
\hline 044 & Stenotomus chrysops & LDPE & 0.89 & -0.10 & 0.0141 & 0.73 & 0.24 & 7 & Morgan and Lohmann (2010) \\
\hline 045 & Stenotomus chrysops & LDPE & 0.53 & 0.23 & 0.0295 & 0.65 & 0.18 & 7 & Morgan and Lohmann (2010) \\
\hline 046 & Stenotomus chrysops & LDPE & 0.52 & 0.36 & 0.0894 & 0.47 & 0.25 & 7 & Morgan and Lohmann (2010) \\
\hline 047 & Peprilus triacanthus & LDPE & 1.22 & -0.33 & 0.0002 & 0.92 & 0.15 & 8 & Morgan and Lohmann (2010) \\
\hline 048 & Peprilus triacanthus & LDPE & 1.05 & 0.33 & 0.0053 & 0.69 & 0.28 & 9 & Morgan and Lohmann (2010) \\
\hline 049 & Peprilus triacanthus & LDPE & 1.05 & 0.31 & 0.0567 & 0.55 & 0.45 & 7 & Morgan and Lohmann (2010) \\
\hline 050 & Peprilus triacanthus & LDPE & 1.12 & 0.12 & 0.0007 & 0.87 & 0.19 & 8 & Morgan and Lohmann (2010) \\
\hline 051 & Peprilus triacanthus & LDPE & 0.88 & 0.07 & 0.0011 & 0.80 & 0.18 & 9 & Morgan and Lohmann (2010) \\
\hline 052 & Peprilus triacanthus & LDPE & 1.10 & -0.20 & 0.0028 & 0.74 & 0.27 & 9 & Morgan and Lohmann (2010) \\
\hline 053 & Peprilus triacanthus & LDPE & 1.02 & 0.02 & 0.0307 & 0.73 & 0.15 & 6 & Morgan and Lohmann (2010) \\
\hline 054 & Peprilus triacanthus & LDPE & 1.04 & -0.58 & 0.0172 & 0.79 & 0.12 & 6 & Morgan and Lohmann (2010) \\
\hline 055 & Peprilus triacanthus & LDPE & 0.80 & -0.15 & 0.1039 & 0.52 & 0.18 & 6 & Morgan and Lohmann (2010) \\
\hline 056 & Peprilus triacanthus & LDPE & 0.53 & 0.78 & 0.1002 & 0.34 & 0.31 & 9 & Morgan and Lohmann (2010) \\
\hline 057 & Peprilus triacanthus & LDPE & 0.57 & 0.22 & 0.1364 & 0.29 & 0.37 & 9 & Morgan and Lohmann (2010) \\
\hline 058 & Abramis brama & $\mathrm{DC} *$ & 0.94 & 0.75 & $<0.0001$ & 0.97 & 0.07 & 7 & Schäfer et al. (2015) \\
\hline 059 & Abramis brama & $\mathrm{DC} *$ & 1.06 & 0.60 & 0.0002 & 0.95 & 0.12 & 7 & Schäfer et al. (2015) \\
\hline 060 & Abramis brama & $\mathrm{DC}^{*}$ & 1.08 & 0.54 & $<0.0001$ & 0.97 & 0.10 & 7 & Schäfer et al. (2015) \\
\hline 061 & Perca fluviatilis & POM & 1.02 & 0.87 & 0.1017 & 0.81 & 0.34 & 4 & Sobek et al. (2014) \\
\hline 062 & Perca fluviatilis & POM & 0.92 & 0.69 & 0.1329 & 0.75 & 0.34 & 4 & Sobek et al. (2014) \\
\hline 063 & Perca fluviatilis & POM & 0.89 & 0.49 & 0.1007 & 0.81 & 0.32 & 4 & Sobek et al. (2014) \\
\hline 064 & Perca fluviatilis & POM & 0.74 & 0.12 & 0.0724 & 0.86 & 0.31 & 4 & Sobek et al. (2014) \\
\hline
\end{tabular}




\section{Table S5. Continued.}

\begin{tabular}{|c|c|c|c|c|c|c|c|c|c|}
\hline Panel & Fish/shellfish species & Polymer & Slope & Intercept & p-value & $r^{2}$ & Sy.x & $\mathbf{n}$ & Reference \\
\hline 065 & Salmo trutta & AlteSil* & 1.08 & 0.99 & 0.0622 & 0.74 & 0.20 & 5 & Allan et al. (2013) \\
\hline 066 & Salmo trutta & AlteSil* & 0.53 & 1.63 & 0.1570 & 0.54 & 0.15 & 5 & Allan et al. (2013) \\
\hline 067 & Salmo trutta & AlteSil* & 0.77 & 1.31 & 0.2094 & 0.46 & 0.26 & 5 & Allan et al. (2013) \\
\hline 068 & Salmo trutta & AlteSil* & 0.75 & 1.23 & 0.1084 & 0.63 & 0.18 & 5 & Allan et al. (2013) \\
\hline 069 & Salmo trutta & AlteSil* & 0.20 & 1.73 & 0.6381 & 0.08 & 0.21 & 5 & Allan et al. (2013) \\
\hline 070 & Salmo trutta & AlteSil* & 1.42 & 0.79 & 0.0953 & 0.66 & 0.32 & 5 & Allan et al. (2013) \\
\hline 071 & Salmo trutta & AlteSil* & 0.72 & 1.25 & 0.2365 & 0.42 & 0.26 & 5 & Allan et al. (2013) \\
\hline 072 & Salmo trutta & AlteSil* & 0.36 & 1.67 & 0.3762 & 0.26 & 0.19 & 5 & Allan et al. (2013) \\
\hline 073 & Danio rerio & POM & 0.79 & 1.06 & $<0.0001$ & 0.76 & 0.29 & 78 & Fadaei et al. (2015) \\
\hline 074 & Morone saxatilis & LDPE & 1.23 & 0.67 & $<0.0001$ & 0.64 & 0.47 & 29 & Khairy et al. (2014) \\
\hline 075 & Hybognathus regius & LDPE & 1.03 & 0.92 & $<0.0001$ & 0.54 & 0.48 & 29 & Khairy et al. (2014) \\
\hline 076 & Dorosoma cepedianum & LDPE & 1.04 & 0.70 & $<0.0001$ & 0.70 & 0.35 & 29 & Khairy et al. (2014) \\
\hline 077 & Fundulus heteroclitus & LDPE & 1.04 & 0.52 & $<0.0001$ & 0.65 & 0.38 & 29 & Khairy et al. (2014) \\
\hline 078 & Menidia menidia & LDPE & 1.04 & 0.98 & $<0.0001$ & 0.63 & 0.40 & 29 & Khairy et al. (2014) \\
\hline 079 & Menidia menidia & LDPE & 1.05 & 1.06 & $<0.0001$ & 0.59 & 0.44 & 29 & Khairy et al. (2014) \\
\hline 080 & Morone americana & LDPE & 1.09 & 0.91 & $<0.0001$ & 0.62 & 0.43 & 29 & Khairy et al. (2014) \\
\hline 081 & Morone saxatilis & LDPE & 1.13 & 0.99 & $<0.0001$ & 0.63 & 0.44 & 29 & Khairy et al. (2014) \\
\hline 082 & Morone americana & LDPE & 1.05 & 1.07 & $<0.0001$ & 0.64 & 0.39 & 29 & Khairy et al. (2014) \\
\hline 083 & Lepomis gibbosus & LDPE & 1.04 & 0.98 & $<0.0001$ & 0.56 & 0.46 & 29 & Khairy et al. (2014) \\
\hline 084 & Hybognathus regius & LDPE & 1.12 & 0.80 & $<0.0001$ & 0.63 & 0.44 & 29 & Khairy et al. (2014) \\
\hline 085 & Fundulus diaphanus & LDPE & 1.11 & 0.74 & $<0.0001$ & 0.68 & 0.38 & 29 & Khairy et al. (2014) \\
\hline 086 & Anguilla rostrata & LDPE & 0.94 & 1.04 & $<0.0001$ & 0.64 & 0.35 & 29 & Khairy et al. (2014) \\
\hline 087 & Anguilla rostrata & LDPE & 1.11 & 1.01 & $<0.0001$ & 0.44 & 0.63 & 29 & Khairy et al. (2014) \\
\hline 088 & Morone saxatilis & LDPE & 1.21 & 0.95 & $<0.0001$ & 0.69 & 0.41 & 29 & Khairy et al. (2014) \\
\hline 089 & Morone saxatilis & LDPE & 1.12 & 0.91 & $<0.0001$ & 0.55 & 0.51 & 29 & Khairy et al. (2014) \\
\hline 090 & Perca fluviatilis & POM & 0.85 & 1.18 & $<0.0001$ & 0.85 & 0.42 & 15 & Sobek et al. (2014) \\
\hline 091 & Perca fluviatilis & POM & 0.76 & 1.34 & 0.0007 & 0.60 & 0.74 & 15 & Sobek et al. (2014) \\
\hline 092 & Perca fluviatilis & POM & 0.67 & 0.76 & 0.0007 & 0.60 & 0.76 & 15 & Sobek et al. (2014) \\
\hline 093 & Callinectes sapidus & LDPE & 1.13 & 1.10 & $<0.0001$ & 0.44 & 0.62 & 29 & Khairy et al. (2014) \\
\hline 094 & Callinectes sapidus & LDPE & 0.97 & 1.59 & 0.0007 & 0.35 & 0.66 & 29 & Khairy et al. (2014) \\
\hline 095 & Homarus americanus & LDPE & 1.20 & 0.22 & 0.0521 & 0.44 & 0.56 & 9 & Morgan and Lohmann (2010) \\
\hline 096 & Homarus americanus & LDPE & 1.05 & 0.53 & 0.0096 & 0.64 & 0.32 & 9 & Morgan and Lohmann (2010) \\
\hline
\end{tabular}




\section{Table S5. Continued.}

\begin{tabular}{|c|c|c|c|c|c|c|c|c|c|}
\hline Panel & Fish species & Polymer & Slope & Intercept & p-value & $r^{2}$ & Sy.x & $\mathbf{n}$ & Reference \\
\hline 097 & Homarus americanus & LDPE & 1.10 & -0.05 & 0.0104 & 0.63 & 0.34 & 9 & Morgan and Lohmann (2010) \\
\hline 098 & Homarus americanus & LDPE & 1.11 & -0.24 & 0.0078 & 0.72 & 0.29 & 8 & Morgan and Lohmann (2010) \\
\hline 099 & Homarus americanus & LDPE & 0.89 & 0.70 & 0.0414 & 0.60 & 0.33 & 7 & Morgan and Lohmann (2010) \\
\hline 100 & Homarus americanus & LDPE & 0.97 & 0.36 & 0.0087 & 0.71 & 0.26 & 8 & Morgan and Lohmann (2010) \\
\hline 101 & Homarus americanus & LDPE & 1.25 & 0.01 & 0.0120 & 0.68 & 0.36 & 8 & Morgan and Lohmann (2010) \\
\hline 102 & Homarus americanus & LDPE & 1.18 & 0.26 & 0.0259 & 0.53 & 0.45 & 9 & Morgan and Lohmann (2010) \\
\hline 103 & Homarus americanus & LDPE & 1.50 & -0.17 & 0.0252 & 0.53 & 0.57 & 9 & Morgan and Lohmann (2010) \\
\hline 104 & Homarus americanus & LDPE & 1.59 & -0.59 & 0.0084 & 0.65 & 0.48 & 9 & Morgan and Lohmann (2010) \\
\hline 105 & Homarus americanus & LDPE & 1.40 & 0.14 & 0.0157 & 0.65 & 0.44 & 8 & Morgan and Lohmann (2010) \\
\hline 106 & Homarus americanus & LDPE & 0.84 & 0.67 & 0.0340 & 0.50 & 0.35 & 9 & Morgan and Lohmann (2010) \\
\hline 107 & Cancer spp. & LDPE & 0.84 & 1.47 & 0.0003 & 0.86 & 0.14 & 9 & Morgan and Lohmann (2010) \\
\hline 108 & Cancer spp. & LDPE & 1.18 & 0.58 & 0.0025 & 0.75 & 0.28 & 9 & Morgan and Lohmann (2010) \\
\hline 109 & Cancer spp. & LDPE & 1.19 & 0.43 & 0.0016 & 0.78 & 0.26 & 9 & Morgan and Lohmann (2010) \\
\hline 110 & Cancer spp. & LDPE & 1.73 & -0.34 & 0.0003 & 0.86 & 0.28 & 9 & Morgan and Lohmann (2010) \\
\hline 111 & Loligo pealei & LDPE & 0.64 & 0.97 & 0.0847 & 0.42 & 0.33 & 8 & Morgan and Lohmann (2010) \\
\hline 112 & Loligo pealei & LDPE & 0.98 & 0.09 & 0.0019 & 0.88 & 0.17 & 7 & Morgan and Lohmann (2010) \\
\hline 113 & Loligo pealei & LDPE & 1.19 & -0.29 & 0.0003 & 0.94 & 0.11 & 7 & Morgan and Lohmann (2010) \\
\hline 114 & Loligo pealei & LDPE & 1.15 & -0.03 & 0.0032 & 0.73 & 0.28 & 9 & Morgan and Lohmann (2010) \\
\hline 115 & Loligo pealei & LDPE & 0.91 & 0.07 & 0.0068 & 0.73 & 0.24 & 8 & Morgan and Lohmann (2010) \\
\hline 116 & Loligo pealei & LDPE & 1.28 & -0.40 & $<0.0001$ & 0.94 & 0.14 & 9 & Morgan and Lohmann (2010) \\
\hline 117 & Loligo pealei & LDPE & 1.07 & -0.11 & $<0.0001$ & 0.94 & 0.11 & 9 & Morgan and Lohmann (2010) \\
\hline 118 & Loligo pealei & LDPE & 1.00 & 0.02 & $<0.0001$ & 0.93 & 0.11 & 9 & Morgan and Lohmann (2010) \\
\hline 119 & Loligo pealei & LDPE & 1.17 & -0.60 & $<0.0001$ & 0.92 & 0.14 & 9 & Morgan and Lohmann (2010) \\
\hline 120 & Loligo pealei & LDPE & 1.15 & 0.01 & $<0.0001$ & 0.90 & 0.15 & 9 & Morgan and Lohmann (2010) \\
\hline 121 & Loligo pealei & LDPE & 1.21 & -0.14 & $<0.0001$ & 0.91 & 0.15 & 9 & Morgan and Lohmann (2010) \\
\hline 122 & Loligo pealei & LDPE & 0.99 & -0.37 & 0.0001 & 0.89 & 0.14 & 9 & Morgan and Lohmann (2010) \\
\hline 123 & Loligo pealei & LDPE & 1.12 & -0.42 & $<0.0001$ & 0.90 & 0.15 & 9 & Morgan and Lohmann (2010) \\
\hline 124 & Ictalurus punctatus & SPMD & -0.02 & 1.58 & 0.9559 & 0.00 & 0.44 & 14 & Echols et al. (2000) \\
\hline 125 & Ictalurus punctatus & SPMD & 0.06 & 1.52 & 0.6896 & 0.02 & 0.19 & 13 & Echols et al. (2000) \\
\hline 126 & Ictalurus punctatus & SPMD & 0.26 & 1.63 & 0.0600 & 0.29 & 0.15 & 13 & Echols et al. (2000) \\
\hline 127 & Ictalurus punctatus & SPMD & 0.20 & 1.73 & 0.0749 & 0.26 & 0.16 & 13 & Echols et al. (2000) \\
\hline 128 & Ictalurus punctatus & SPMD & 0.60 & 1.63 & $<0.0001$ & 0.86 & 0.20 & 16 & Echols et al. (2000) \\
\hline
\end{tabular}


Table S5. Continued.

\begin{tabular}{|c|c|c|c|c|c|c|c|c|c|}
\hline Panel & Fish species & Polymer & Slope & Intercept & p-value & $r^{2}$ & Sy.x & $\mathbf{n}$ & Reference \\
\hline 129 & Ictalurus punctatus & SPMD & 0.91 & 1.46 & 0.0151 & 0.81 & 0.68 & 6 & Gale et al. (1997) \\
\hline 130 & Ictalurus punctatus & SPMD & 0.94 & 1.33 & 0.0105 & 0.84 & 0.61 & 6 & Gale et al. (1997) \\
\hline 131 & Ictalurus punctatus & SPMD & 1.05 & 1.45 & 0.0242 & 0.86 & 0.57 & 5 & Gale et al. (1997) \\
\hline 132 & Ictalurus punctatus & SPMD & 1.03 & 1.53 & 0.0343 & 0.82 & 0.63 & 5 & Gale et al. (1997) \\
\hline 133 & Ictalurus punctatus & SPMD & 1.01 & 1.18 & 0.0213 & 0.87 & 0.51 & 5 & Gale et al. (1997) \\
\hline 134 & Cyprinus carpio & SPMD & -1.32 & 3.09 & 0.2390 & 0.87 & 0.07 & 3 & Verweij et al. (2004) \\
\hline 135 & Cyprinus carpio & SPMD & 0.39 & 0.70 & 0.0266 & 0.75 & 0.07 & 6 & Verweij et al. (2004) \\
\hline 136 & Cyprinus carpio & SPMD & 0.64 & 0.23 & 0.5691 & 0.19 & 0.21 & 4 & Verweij et al. (2004) \\
\hline 137 & Cyprinus carpio & SPMD & -0.43 & 1.84 & 0.2166 & 0.89 & 0.06 & 3 & Verweij et al. (2004) \\
\hline 138 & Cyprinus carpio & SPMD & -0.48 & 2.17 & 0.2537 & 0.56 & 0.11 & 4 & Verweij et al. (2004) \\
\hline 139 & Cyprinus carpio & SPMD & 2.29 & -1.96 & 0.2358 & 0.87 & 0.19 & 3 & Verweij et al. (2004) \\
\hline 140 & Cyprinus carpio & SPMD & 1.13 & -0.62 & 0.0273 & 0.95 & 0.05 & 4 & Verweij et al. (2004) \\
\hline 141 & Cyprinus carpio & SPMD & -3.17 & 6.03 & 0.5568 & 0.41 & 0.33 & 3 & Verweij et al. (2004) \\
\hline 142 & Cyprinus carpio & SPMD & 0.28 & 1.21 & 0.6479 & 0.12 & 0.13 & 4 & Verweij et al. (2004) \\
\hline 143 & Cyprinus carpio & SPMD & 0.97 & -0.15 & $<0.0001$ & 0.67 & 0.56 & 21 & Wang et al. (2002) \\
\hline 144 & Cyprinus carpio & SPMD & 0.97 & 0.31 & $<0.0001$ & 0.88 & 0.30 & 21 & Wang et al. (2002) \\
\hline
\end{tabular}

DC = Dow Corning 1-2577 conformal coating; LDPE = low density polyethylene; PDMS = polydimethylsiloxane; POM = polyoxymethylene; SPMDs = semipermeable membrane device. 
In four publications by Gale et al. (1997), ${ }^{13}$ Echols et al. (2000), ${ }^{19}$ Wang et al. (2002), ${ }^{21}$ and Verweij et al. (2004), ${ }^{18}$ PCBs were measured in fish as well as by SPMDs deployed in the water column (Table S2). SPMDs consist of LDPE tubing containing the synthetic lipid triolein, ${ }^{22,23}$ and the biphasic nature of this sampler is different from simpler monophasic polymeric samplers, which are the primary focus of this review. In addition, SPMDs are not routinely deployed in sediments ${ }^{24}$ which limits their relevance to this analysis.

In the studies by Gale et al. (1997) ${ }^{13}$ and Echols et al. (2000), ${ }^{19}$ PCB congeners were measured in channel catfish (Ictalurus punctatus) and in SPMDs deployed in situ in the water column of Saginaw River (MI, USA). Both the caged fish and the SPMDs were deployed in the water column for 28 days. In the study by Wang et al. (2002), ${ }^{21}$ PCB congeners were measured in carp (Cyprinus carpio) and in SPMDs deployed in situ in the water column of Huaihe River (China). The SPMDs were deployed in the water column for 28 days and the fish were indigenous and wild caught. In the study by Verweij et al. (2004), ${ }^{18}$ PCB congeners were measured in carp
(Cyprinus carpio) and in SPMDs deployed in situ in the water column at nine locations in Amsterdam and surroundings (The Netherlands); a city canal, several ditches (treated as one location), two harbors, a lake, and four river sites. Both the caged fish and the SPMDs were deployed in the water column for 4 weeks.

From these four publications, ten regressions were produced linking $C_{L}$ in channel catfish to $C_{P}$ (SPMD equilibrium assumed) while 11 regressions were produced linking $C_{L}$ in carp to $C_{P}$ (Figure S2, panes 124-144, Table S5). The SPMD equilibrium was assumed in the study by Wang et al. (2002) $)^{21}$ and inferred via performance reference compounds in the study by Verweij et al. (2004). ${ }^{18}$ The average $r^{2}$ value of the 21 regressions was 0.61 (range: $0.00027-0.95, n=21$ ). A total of 10 regressions (48\%) were statistically significant $(p<0.05)$. For comparison, the average $r^{2}$ value of regressions linking $C_{L}$ in fish and $C_{p}$ based on deployment of monophasic samplers in situ in the water column was 0.59 (range: $0.08-0.85, n=28$ ). Thus, the average $r^{2}$ values were in line when comparing regressions based on SPMD data and regressions based on the monophasic sampler data, despite the much wider range in $r^{2}$ values for the regressions based on SPMD data and the relatively low percentage of statistically significant regressions ( $48 \%$ versus $71 \%$ ).

Most of the 21 regressions based on SPMD data were above the (gray) range of \pm a factor of 10 from the hypothetical 1:1 relationship (Figure S2, panels 124-144). Still, the vast majority of the regressions were within a factor of 100 from the hypothetical 1:1 relationship. However, it should be noted that several regressions had a negative slope, meaning that increasing PCB concentrations in fish were not reflected by increasing $P C B$ concentrations in SPMD. Based on this observation, the lower percentage of statistically significant regressions ( $p$ $>0.05$ ), and the extremely wide range in $r^{2}$ values, it is unadvisable to use SPMD polymeric sampling as a tool for predicting the bioaccumulation of PCBs by fish (and most likely other aquatic organisms). 


\begin{tabular}{|c|c|c|c|c|c|c|c|c|c|}
\hline Variable & Category & Average $r^{2}$ & Range & Average Sy.x & Range & $n^{\text {a) }}$ & $n(p<0.05)^{b)}$ & $\begin{array}{c}\text { Average } \\
\log \left(C_{L} / C_{P}\right) \pm S D\end{array}$ & $n^{c)}$ \\
\hline \multirow[t]{3}{*}{ Polymer } & Silicone & 0.80 & $0.26-0.97$ & 0.29 & $0.07-0.53$ & 14 & 11 & $0.39 \pm 0.43$ & 94 \\
\hline & POM & 0.77 & $0.62-0.86$ & 0.34 & $0.31-0.37$ & 5 & 1 & $0.57 \pm 0.47$ & 95 \\
\hline & LDPE & 0.74 & $0.29-0.97$ & 0.26 & $0.08-0.55$ & 45 & 40 & $0.55 \pm 0.52$ & 692 \\
\hline \multirow[t]{3}{*}{ Equilibrium } & Assumed & 0.76 & $0.29-0.97$ & 0.21 & $0.08-0.45$ & 33 & 24 & $0.16 \pm 0.47$ & 244 \\
\hline & Inferred & 0.71 & $0.57-0.85$ & 0.37 & $0.29-0.55$ & 16 & 16 & $0.76 \pm 0.42$ & 464 \\
\hline & Confirmed & 0.79 & $0.26-0.97$ & 0.29 & $0.07-0.53$ & 15 & 12 & $0.45 \pm 0.46$ & 173 \\
\hline \multirow[t]{4}{*}{ Trophic level } & $<3.0$ & 0.68 & $0.65-0.71$ & 0.39 & $0.37-0.40$ & 3 & 3 & $0.65 \pm 0.40$ & 87 \\
\hline & $3.0-3.4$ & 0.80 & $0.62-0.97$ & 0.26 & $0.07-0.38$ & 12 & 12 & $0.70 \pm 0.41$ & 288 \\
\hline & $3.5-3.9$ & 0.73 & $0.26-0.97$ & 0.25 & $0.08-0.55$ & 38 & 30 & $0.25 \pm 0.52$ & 353 \\
\hline & $\geq 4.0$ & 0.82 & $0.73-0.94$ & 0.32 & $0.22-0.41$ & 11 & 7 & $0.80 \pm 0.42$ & 153 \\
\hline \multirow[t]{4}{*}{ Habitat } & Marine & 0.81 & $0.75-0.86$ & 0.29 & $0.16-0.34$ & 5 & 1 & $0.75 \pm 0.25$ & 23 \\
\hline & Riverine & 0.75 & $0.57-0.97$ & 0.33 & $0.07-0.55$ & 20 & 20 & $0.75 \pm 0.41$ & 485 \\
\hline & Lacustrine & 0.75 & $0.26-0.94$ & 0.36 & $0.22-0.53$ & 10 & 7 & $0.26 \pm 0.45$ & 66 \\
\hline & Estuarine & 0.76 & $0.29-0.97$ & 0.20 & $0.08-0.45$ & 29 & 24 & $0.12 \pm 0.46$ & 228 \\
\hline \multirow[t]{5}{*}{ Migration } & Oceanodromous & 0.76 & $0.29-0.97$ & 0.20 & $0.08-0.45$ & 32 & 27 & $0.28 \pm 0.52$ & 293 \\
\hline & Anadromous & 0.78 & $0.65-0.91$ & 0.34 & $0.29-0.41$ & 12 & 8 & $0.80 \pm 0.40$ & 226 \\
\hline & Potamodromous & 0.91 & $0.71-0.97$ & 0.20 & $0.07-0.38$ & 7 & 7 & $0.61 \pm 0.39$ & 71 \\
\hline & Catadromous & 0.63 & $0.26-0.83$ & 0.43 & $0.32-0.55$ & 8 & 5 & $0.55 \pm 0.55$ & 96 \\
\hline & Nonmigratory & 0.68 & $0.60-0.80$ & 0.37 & $0.30-0.41$ & 5 & 5 & $0.57 \pm 0.44$ & 195 \\
\hline
\end{tabular}




\begin{tabular}{|c|c|c|c|c|c|c|c|c|c|}
\hline Variable & Category & Average $r^{2}$ & Range & Average Sy.x & Range & $n^{\text {a) }}$ & $\mathrm{n}(\mathrm{p}<0.05)^{\mathrm{b})}$ & $\begin{array}{c}\text { Average } \\
\log \left(C_{L} / C_{P}\right) \pm S D\end{array}$ & $\mathrm{n}^{\mathrm{c})}$ \\
\hline \multirow[t]{3}{*}{ Polymer } & Silicone & 0.47 & $0.08-0.74$ & 0.22 & $0.15-0.32$ & 8 & 0 & $1.16 \pm 0.24$ & 40 \\
\hline & POM & 0.70 & $0.60-0.85$ & 0.56 & $0.29-0.76$ & 4 & 4 & $0.98 \pm 0.59$ & 123 \\
\hline & LDPE & 0.62 & $0.44-0.70$ & 0.43 & $0.35-0.63$ & 16 & 16 & $1.00 \pm 0.46$ & 464 \\
\hline \multirow[t]{2}{*}{ Equilibrium } & Assumed & 0.70 & $0.60-0.85$ & 0.56 & $0.29-0.76$ & 4 & 4 & $0.98 \pm 0.59$ & 123 \\
\hline & Inferred & 0.57 & $0.08-0.74$ & 0.36 & $0.15-0.63$ & 24 & 16 & $1.02 \pm 0.45$ & 504 \\
\hline \multirow{4}{*}{ Trophic level } & $<3.0$ & 0.62 & $0.54-0.70$ & 0.42 & $0.35-0.48$ & 3 & 3 & $0.89 \pm 0.42$ & 87 \\
\hline & $3.0-3.4$ & 0.55 & $0.08-0.76$ & 0.30 & $0.15-0.46$ & 15 & 7 & $0.98 \pm 0.40$ & 292 \\
\hline & $3.5-3.9$ & 0.58 & $0.44-0.65$ & 0.46 & $0.35-0.63$ & 3 & 3 & $0.90 \pm 0.52$ & 87 \\
\hline & $\geq 4.0$ & 0.65 & $0.55-0.85$ & 0.54 & $0.41-0.76$ & 7 & 7 & $1.18 \pm 0.56$ & 161 \\
\hline \multirow[t]{2}{*}{ Habitat } & Marine & 0.68 & $0.60-0.85$ & 0.64 & $0.42-0.76$ & 3 & 3 & $1.36 \pm 0.74$ & 45 \\
\hline & Riverine & 0.57 & $0.08-0.74$ & 0.36 & $0.15-0.63$ & 24 & 16 & $1.02 \pm 0.45$ & 504 \\
\hline \multirow[t]{5}{*}{ Migration } & Oceanodromous & 0.61 & $0.59-0.63$ & 0.42 & $0.40-0.44$ & 2 & 2 & $1.08 \pm 0.41$ & 58 \\
\hline & Anadromous & 0.57 & $0.08-0.85$ & 0.37 & $0.15-0.76$ & 18 & 10 & $1.11 \pm 0.49$ & 288 \\
\hline & Potamodromous & 0.56 & - & 0.46 & - & 1 & 1 & $1.04 \pm 0.45$ & 29 \\
\hline & Catadromous & 0.54 & $0.44-0.64$ & 0.49 & $0.35-0.63$ & 2 & 2 & $1.06 \pm 0.51$ & 58 \\
\hline & Nonmigratory & 0.65 & $0.54-0.76$ & 0.39 & $0.29-0.48$ & 5 & 5 & $0.81 \pm 0.40$ & 194 \\
\hline
\end{tabular}




\section{Details on the five key experimental variables}

Type of polymer: For simplicity and reporting purposes, three commercial silicones (i.e., AlteSil, DC, and PDMS) were grouped for this analysis. For the application of DC and PDMS in equilibrium sampling, glass jars were internally coated with multiple $\mu \mathrm{m}$-thin coatings $(<10 \mu \mathrm{m})$ of the polymers, which enabled full equilibration of the PCBs between sediment interstitial water and the polymer. ${ }^{4,5,16,20}$ After sampling, equilibrium concentrations in the silicone were confirmed by PCB to polymer mass regression analyses. ${ }^{4,5,16,20}$ The polymers LDPE and POM were applied as sheets and strips for ex situ passive sampling of sediment interstitial water as well as for in situ passive sampling in the water column in the field. ${ }^{14,15,17}$ In addition to LDPE and POM, the silicone-based polymer AlteSil was also applied as strips for in situ sampling of PCBs in the water column of River Alna (Norway). ${ }^{11}$ As mentioned, equilibration of more hydrophobic compounds between (sediment interstitial) water and the polymer is rarely achieved in passive sampling, and the equilibrium is most often assumed ${ }^{15,17}$ or inferred using performance reference compounds (PRCs) amended into the polymer before deployment. ${ }^{11,14}$

Polymer equilibrium status: Equilibrium concentrations in the sampling polymer are confirmed when following the equilibrium sampling approach, whereas they are most often assumed or inferred following the passive sampling approach. Equilibrium polymer concentrations form a common benchmark that enables quantitative analysis and comparison of data resulting from different polymeric sampling approaches, with different types and thicknesses of polymer and different deployment strategies. Underestimations of polymer equilibration relative to the sampling medium might influence the strength of the correlation between lipidnormalized concentrations and (equilibrium) polymer concentration, as the degree of disequilibrium most often increases with increasing compound hydrophobicity. ${ }^{25}$ Thus, less hydrophobic compounds might be at equilibrium between the polymer and the sampling medium whereas highly hydrophobic compounds may be very far from equilibrium. ${ }^{26}$ Moreover, polymers that are underequilibrated will underestimate bioaccumulation relative to the lipid-normalized concentration in fish. 
Fish tropic level: The vast majority of the fish were wild caught before, during, or after collection of sediment for ex situ polymeric sampling of sediment interstitial water or deployment of samplers in the water column for in situ passive sampling. The two exceptions were zebrafish (Danio rerio) used in mesocosm experiments with sediment from Grasse River (NY, USA) ${ }^{12}$ and trout (Salmo trutta) which were caged and exposed in the River Alna (Norway) alongside AlteSil silicone strips for passive sampling of PCBs in the water column. ${ }^{11}$ The trophic level for each of the 20 fish species was assigned using the online database FishBase (Table S4). In FishBase, ${ }^{27}$ the trophic level (TL) of a given fish is calculated based on dietary studies. Herbivorous fish are primary consumers (TL 2), carnivorous fish that eat herbivorous fish are secondary consumers (TL 3), while carnivorous fish that eat other carnivorous fish are tertiary consumers (TL 4). Of the 20 fish species, two species (Hybognathus regius and Dorosoma cepedianum) were classified as primary consumers, while four species (Sander lucioperca, Esox lucius, Perca fluviatilis, and Morone saxatilis) were classified as tertiary consumers. The remaining 14 fish species were classified as secondary consumers, and most data was collected for this group of fish. The lowest trophic level fish in the data set was Hybognathus regius ( $T L=2.0)$, while the highest trophic level fish was Morone saxatilis ( $T L=4.7)$.

Fish habitat: The sediment for ex situ polymeric sampling was collected within the same (larger) area from which the fish were caught. In the studies with in situ passive sampling, the passive samplers were in most cases deployed in the water column close to where the fish were caught. The two exceptions were the mesocosm study ${ }^{12}$ and the study with caged fish. ${ }^{11}$ The studies were conducted in four different habitats (i.e., estuarine, lacustrine, riverine, and marine) at locations in Europe and in the United States. The data set was dominated by data from Lower Passaic River (NJ, USA), ${ }^{14}$ River Alna (Norway), ${ }^{11}$ River Elbe (Germany), ${ }^{16}$ and Grasse River (NY, USA). ${ }^{12}$ Data from Narragansett Bay (RI, USA) ${ }^{15}$ an estuary, dominated the remaining part of the data set, which also contained a substantial amount of data from two locations in the Swedish part of the Baltic Sea (the Gulf of Bothnia ${ }^{17}$ and the Stockholm Archipelago ${ }^{20}$ ) and from Lake Ången (Sweden). ${ }^{4,5}$ 
Fish migratory status: The migratory status for each of the 20 fish species was assigned using the online database FishBase ${ }^{27}$ (Table S4). Based on this assignment, the fish were grouped into five categories. First, four species (Clupea harengus, Menidia menidia, Peprilus triacanthus, and Stenotomus chrysops) were classified as oceanodromous, which denotes fish living and migrating exclusively in salt water. Second, five species (Dorosoma cepedianum, Morone americana, Morone saxatilis, Perca fluviatilis, and Salmo trutta) were classified as anadromous, which denotes fish living in salt water and ascending rivers from the sea for breeding. Third, five species (Abramis brama, Esox Lucius, Lepomis gibbosus, Rutilus rutilus, and Sander lucioperca) were classified as potamodromous, which denotes fish living and migrating exclusively in fresh water. Forth, two species (Anguilla anguilla and Anguilla rostrate) were classified as catadromous, which denotes fish living in fresh water and going to the sea to spawn. Finally, four species (Danio rerio, Fundulus diaphanus, Fundulus heteroclitus, and Hybognathus regius) were classified as nonmigratory. 


\section{References}

1. Mayer, P.; Vaes, W. H.; Wijnker, F.; Legierse, K. C.; Kraaij, R.; Tolls, J.; Hermens, J. L., Sensing dissolved sediment porewater concentrations of persistent and bioaccumulative pollutants using disposable solid-phase microextraction fibers. Environ. Sci. Technol. 2000, 34, 5177-5183.

2. Reichenberg, F.; Mayer, P., Two complementary sides of bioavailability: Accessibility and chemical activity of organic contaminants in sediments and soils. Environ. Toxicol. Chem. 2006, 25, 1239-1245.

3. Mäenpää, K.; Leppänen, M. T.; Reichenberg, F.; Figueiredo, K.; Mayer, P., Equilibrium sampling of persistent and bioaccumulative compounds in soil and sediment: Comparison of two approaches to determine equilibrium partitioning concentrations in lipids. Environ. Sci. Technol. 2011, 45, 1041-1047.

4. Jahnke, A.; Mayer, P.; McLachlan, M. S.; Wickström, H.; Gilbert, D.; MacLeod, M., Silicone passive equilibrium samplers as 'chemometers' in eels and sediments of a Swedish lake. Environ. Sci.: Processes Impacts 2014, 16, 464-472.

5. Jahnke, A.; MacLeod, M.; Wickström, H. k.; Mayer, P., Equilibrium sampling to determine the thermodynamic potential for bioaccumulation of persistent organic pollutants from sediment. Environ. Sci. Tech. 2014, 48, 11352-11359.

6. Gschwend, P. M.; MacFarlane, J. K.; Reible, D. D.; Lu, X.; Hawthorne, S. B.; Nakles, D. V.; Thompson, T., Comparison of polymeric samplers for accurately assessing PCBs in pore waters. Environ. Toxicol. Chem. 2011, 30, 1288-1296.

7. Fernandez, L. A.; Lao, W.; Maruya, K. A.; Burgess, R. M., Calculating the diffusive flux of persistent organic pollutants between sediments and the water column on the Palos Verdes Shelf Superfund Site using polymeric passive samplers. Environ. Sci. Technol. 2014, 48, 3925-3934.

8. $\quad$ Burgess, R. M.; Lohmann, R.; Schubauer-Berigan, J. P.; Reitsma, P.; Perron, M. M.; Lefkovitz, L.; Cantwell, M. G., Application of passive sampling for measuring dissolved concentrations of organic contaminants in the water column at three marine superfund sites. Environ. Toxicol. Chem. 2015, 34, 1720-1733.

9. $\quad$ Fernandez, L. A.; Harvey, C. F.; Gschwend, P. M., Using performance reference compounds in polyethylene passive samplers to deduce sediment porewater concentrations for numerous target chemicals. Environ. Sci. Technol. 2009, 43, 8888-8894.

10. Ghosh, U.; Driscoll, S. K.; Burgess, R. M.; Jonker, M. T.; Reible, D.; Gobas, F.; Choi, Y.; Apitz, S. E.; Maruya, K. A.; Gala, W. R.; Mortimer, M.; Beegan, C., Passive sampling methods for contaminated sediments: Practical guidance for selection, calibration, and implementation. Integr. Environ. Assess. Manag. 2014, 10, 210-223.

11. Allan, I. J.; Bæk, K.; Haugen, T. O.; Hawley, K. L.; Høgfeldt, A. S.; Lillicrap, A. D., In vivo passive sampling of nonpolar contaminants in brown trout (Salmo trutta). Environ. Sci. Technol. 2013, 47, 11660-11667.

12. Fadaei, H.; Watson, A.; Place, A.; Connolly, J.; Ghosh, U., Effect of PCB bioavailability changes in sediments on bioaccumulation in fish. Environ. Sci. Technol. 2015, 49, 12405-12413.

13. Gale, R. W.; Huckins, J. N.; Petty, J. D.; Peterman, P. H.; Williams, L. L.; Morse, D.; Schwartz, T. R.; Tillitt, D. E., Comparison of the uptake of dioxin-like compounds by caged channel catfish and semipermeable membrane devices in the Saginaw River, Michigan. Environ. Sci. Technol. 1997, 31, 178-187. 
14. Khairy, M. A.; Weinstein, M. P.; Lohmann, R., Trophodynamic behavior of hydrophobic organic contaminants in the aquatic food web of a tidal river. Environ. Sci. Technol. 2014, 48, 12533-12542.

15. Morgan, E. J.; Lohmann, R., Dietary uptake from historically contaminated sediments as a source of PCBs to migratory fish and invertebrates in an urban estuary. Environ. Sci. Technol. 2010, 44, 5444-5449.

16. Schäfer, S.; Antoni, C.; Möhlenkamp, C.; Claus, E.; Reifferscheid, G.; Heininger, P.; Mayer, P., Equilibrium sampling of polychlorinated biphenyls in River Elbe sediments - Linking bioaccumulation in fish to sediment contamination. Chemosphere 2015, 138, 856-862.

17. Sobek, A.; Wiberg, K.; Sundqvist, K.; Haglund, P.; Jonsson, P.; Cornelissen, G., Coastal sediments in the Gulf of Bothnia as a source of dissolved PCDD/Fs and PCBs to water and fish. Sci. Tot. Environ. 2014, 487, 463-470.

18. Verweij, F.; Booij, K.; Satumalay, K.; van der Molen, N.; van der Oost, R., Assessment of bioavailable PAH, PCB and OCP concentrations in water, using semipermeable membrane devices (SPMDs), sediments and caged carp. Chemosphere 2004, 54, 1675-1689.

19. Echols, K. R.; Gale, R. W.; Schwartz, T. R.; Huckins, J. N.; Williams, L. L.; Meadows, J. C.; Morse, D.; Petty, J. D.; Orazio, C. E.; Tillitt, D. E., Comparing polychlorinated biphenyl concentrations and patterns in the Saginaw River using sediment, caged fish, and semipermeable membrane devices. Environ. Sci. Technol. 2000, 34, 4095-4102.

20. Jahnke, A.; Mayer, P.; McLachlan, M. S., Sensitive equilibrium sampling to study polychlorinated biphenyl disposition in Baltic Sea sediment. Environ. Sci. Technol. 2012, 46, 10114-10122.

21. Wang, Z.; Wang, Y.; Ma, M.; Lu, Y.; Huckins, J., Use of triolein-semipermeable membrane devices to assess the bioconcentration and sediment sorption of hydrophobic organic contaminants in the Huaihe River, China. Environ. Toxicol. Chem. 2002, 21, $2378-2384$.

22. Huckins, J. N.; Tubergen, M. W.; Manuweera, G. K., Semipermeable membrane devices containing model lipid: A new approach to monitoring the bioavailability of lipophilic contaminants and estimating their bioconcentration potential. Chemosphere 1990, 20, 533-552.

23. Huckins, J. N.; Manuweera, G. K.; Petty, J. D.; Mackay, D.; Lebo, J. A., Lipid-containing semipermeable membrane devices for monitoring organic contaminants in water. Environ. Sci. Technol. 1993, 27, 2489-2496.

24. Joyce, A. S.; Portis, L. M.; Parks, A. N.; Burgess, R. M., Evaluating the relationship between equilibrium passive sampler uptake and aquatic organism bioaccumulation. Environ. Sci. Technol. 2016, 50, 11437-11451.

25. Schmidt, S. N.; Wang, A. P.; Gidley, P. T.; Wooley, A. H.; Lotufo, G. R.; Burgess, R. M.; Ghosh, U.; Fernandez, L. A.; Mayer, P., Cross validation of two partitioning-based sampling approaches in mesocosms containing PCB contaminated field sediment, biota, and activated carbon amendment. Environ. Sci. Technol. 2017, 51, 9996-10004.

26. Joyce, A. S.; Burgess, R. M., Using performance reference compounds to compare mass transfer calibration methodologies in passive samplers deployed in the water column. Environ. Toxicol. Chem. 2018, 37, 2089-2097.

27. Froese, R.; Pauly, D., Editors. 2019. FishBase. World Wide Web electronic publication. www.fishbase.org, version (04/2019). Last accessed August 11, 2019. 